\title{
Dual Mode Microwave Microfluidic Sensor for Temperature Variant Liquid Characterization
}

\author{
Ali A. Abduljabar, Nicholas Clark, Jonathan Lees, and Adrian Porch
}

\begin{abstract}
A dual mode, microstrip, microfluidic sensor was designed, built, and tested, which has the ability to measure a liquid's permittivity at $2.5 \mathrm{GHz}$ and, simultaneously, compensate for temperature variations. The active liquid volume is small, only around $4.5 \mu \mathrm{L}$. The sensor comprises two quarter ring microstrip resonators, which are excited in parallel. The first of these is a microfluidic sensor whose resonant frequency and quality factor depend on the dielectric properties of a liquid sample. The second is used as a reference to adjust for changes in the ambient temperature. To validate this method, two liquids (water and chloroform) have been tested over a temperature range from $23{ }^{\circ} \mathrm{C}$ to $35{ }^{\circ} \mathrm{C}$, with excellent compensation results.
\end{abstract}

Index Terms-Dielectric measurement, microwave microfluidic sensor, temperature sensor.

\section{INTRODUCTION}

$\mathbf{C}$ HARACTERIZATION of a liquid's dielectric properties using microwave techniques, quantified by its complex permittivity, has many advantages over other methods. These include low cost, noninvasiveness, high speed (effectively real time), and the ability to be miniaturized, so opening up labon-chip applications. Much research has been undertaken in the general area of liquid characterization. For example, a microfabricated microwave resonator is proposed [1] for the characterisation of liquids in biochemical applications, where nanoliter volumes of aqueous solutions have been located on the top surface of the planar resonator. A microwave microfluidic sensor inspired by metamaterial concepts [2] comprises a complementary split-ring resonator with a microchannel containing the liquid under test. Split-ring resonators fabricated using silver-coated copper wire for microwave microfluidic sensing have been presented in [3]. A microstrip split-ring resonator sensor, this time with two gaps, has been described in [4] to characterize the dielectric properties of common solvents within a microfluidic environment.

The two-gap microwave sensor was applied in [5] characterizing the dielectric and kinematic properties of a microfluidic segmented flow, including segment volumes and velocities. A very sensitive and tunable radio-frequency circuit was

Manuscript received October 28, 2016; revised December 6, 2016; accepted December 10, 2016. The work of N. Clark was supported by an EPSRC iCASE studentship EP/L505390/1.

A. A. Abduljabar is with the Department of Electrical Engineering, College of Engineering, University of Basrah, 61001 Basrah, Iraq (e-mail: aliaiq76@gmail.com).

N. Clark, J. Lees, and A. Porch are with the School of Engineering, Cardiff University, Cardiff CF24 3AA, U.K. (e-mail: clarkns@ cardiff.ac.uk; leesJ2@cardiff.ac.uk; porcha@cardiff.ac.uk).

Color versions of one or more of the figures in this paper are available online at http://ieeexplore.ieee.org.

Digital Object Identifier 10.1109/TMTT.2016.2647249 presented in [6] for characterizing the dielectric properties of aqueous solutions, where two quadrature hybrids are used for destructive interference to eliminate the presence of the probing signals at the two measurement ports. A highly sensitive sensor for precise permittivity change detection of liquids was presented in [7] based on a planar half-wavelength microstrip line resonator beneath a microfluidic container. A coaxial resonator method was used in [8] that utilized the spatial separation of the electric and magnetic field antinodes (by quarter of a wavelength) for simultaneous measurement of the permittivity and permeability of liquid systems within a small quartz capillary.

However, a common problem in all of these microwave microfluidic sensors is the adverse effect of temperature variation, as the liquid permittivity is often very sensitive to temperature changes (water being a prime example) which yields inaccurate measurements involving large systematic errors. Several works have focussed on solving this problem. In [9], the error frequency shift of a resonator due to temperature dependence of the sample's dielectric properties was determined by using reference measurements in which the resonator does not need cooling. Another idea was proposed in [10] and [11], where an unperturbed mode can be used to assess and correct for very small temperature changes. However, there is still a small influence of the sample on this mode, which complicates the correction process.

A new, versatile sensor is proposed in this paper whose novelty lies in it having a reference resonator, spectrally separated from the measurement mode. Its reference resonant frequency is insensitive to the presence of the dielectric sample, more so than the aforementioned case [10], [11] and so can be used to monitor the temperature changes and hence to adjust ("calibrate") the dielectric measurements accordingly. Such separation of the spectral responses of the measurement and reference sensors is accomplished by the design, which uses different resonators for each function, while allowing for simultaneous excitation. The block diagram of the sensor is shown in Fig. 1, which illustrates the principle of using the two resonators; one is for liquid sample characterization (i.e., of a liquid in a capillary), the other for temperature calibration. We restrict our experimental temperature range to $23{ }^{\circ} \mathrm{C}$ to $35{ }^{\circ} \mathrm{C}$, typical for lab-on-chip applications at "room temperature."

The rest of this paper is arranged as follows. In Section II, the relevant microwave theory and concepts, the temperature dependence of liquids and the microstip resonators adopted, and use of a LabVIEW interface for data collection are presented. We label this type of sensor "DMS," i.e., a dual-mode 


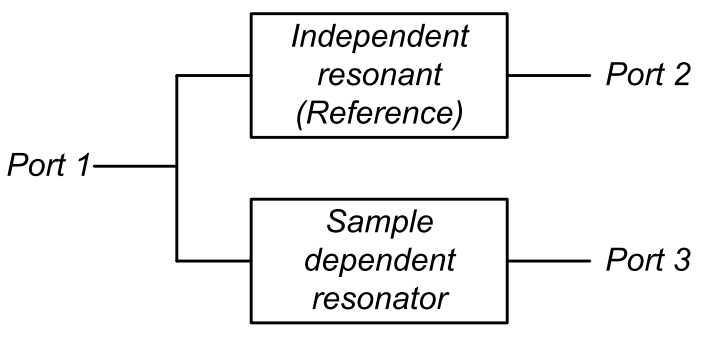

Fig. 1. Schematic of the DMS using two resonators.

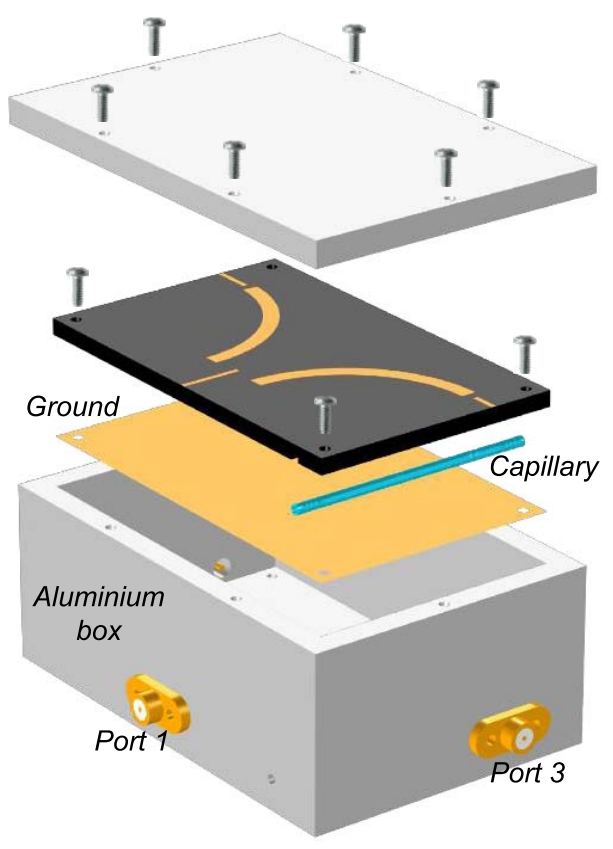

Fig. 2. Schematic of the dual mode microstrip sensor DMS inside its aluminum package.

mircostrip sensor. Descriptions of the DMS design, COMSOL simulation, and fabrication are presented in Section III. The experimental results are shown and discussed in Section IV, with final conclusions and future ideas presented in Section V.

\section{A. Resonance Perturbation}

\section{THEORY AND CONCEPTS}

A quarter ring microstrip resonator is adopted for the DMS sensor, as shown in Fig. 2. In this structure, the electric field energy is stored predominantly in the dielectric substrate. If the sample capillary is filled with a liquid sample and is inserted within the dielectric at the microstrip's end (as shown in Fig. 2), a material-dependent perturbation will occur which causes a downwards shift $\Delta f$ in the resonant frequency $f$ and reduction in the unloaded quality factor $Q$ [12]. Since the sample axis is aligned perpendicular to the applied electric field, the observed perturbations on the microstrip resonator are small and so we can apply first-order perturbation theory [3] to understand how the sample affects the resonator

$$
\begin{gathered}
\Delta f=f_{1}-f_{0} \approx-f_{0} \operatorname{Re}\left(\frac{\Delta p E_{0}^{*}}{4 U}\right) \\
\Delta\left(\frac{1}{Q}\right)=\frac{1}{Q_{1}}-\frac{1}{Q_{0}} \approx-\frac{\Delta Q}{Q_{0}^{2}} \approx-\operatorname{Im}\left(\frac{\Delta p E_{0}^{*}}{2 U}\right) .
\end{gathered}
$$

Here, the subscripts " 0 " and "1" refer to the cases when the capillary is empty and filled with liquid, respectively. $\Delta p$ is the change in dipole moment of the capillary when filled with liquid, $E_{0}$ is the applied electric field, and $U$ is the time averaged stored energy of the resonant mode. A direct link can be made between $\Delta p$ and the complex permittivity of the liquid [4], thus enabling permittivity to be extracted from simultaneous measurements of $f$ and $Q$, corrected for temperature using the reference mode of the DMS.

\section{B. Temperature Dependence}

Changes in ambient temperature will affect both resonant frequency and $Q$ of a resonant microstrip line, in addition to the dielectric properties of the liquid under investigation. Considering the latter quantity first, for polar liquids such as those in our study, the complex permittivity at a certain frequency $f$ is well-described by the Debye model [13]-[15]

$$
\begin{aligned}
& \varepsilon(f, T)=\varepsilon_{\infty}(T)+\frac{\varepsilon_{s}(T)-\varepsilon_{\infty}(T)}{1+j 2 \pi f \tau(T)} \\
& \varepsilon(f, T)=\varepsilon_{1}(f, T)-j \varepsilon_{2}(f, T)
\end{aligned}
$$

where $\varepsilon_{s}$ and $\varepsilon_{\infty}$ are the values of permittivity in very low $(\omega \tau \ll 1)$ and very high $(\omega \tau \gg 1)$ frequency limits, respectively, and $\tau$ is the dielectric relaxation time. $T$ is the temperature. As temperature increases, both real and imaginary parts of the liquid permittivity decrease with temperature. The impact on the microstrip resonator [via (1) and (2)] is two-fold. There will be an increase in the resonant frequency (associated with a reduction in the dipole moment of the sample) and an increase in $Q$ owing to the reduced loss.

Consider next the effects of increased temperature on the empty (host) microstrip resonator. The resonant frequency is related to the length of the strip by the approximate relationship [16]

$$
f=n c / 2 l \sqrt{\varepsilon}_{\text {eff }}
$$

where $l$ is the line length and $n$ is the integer mode number (we use $n=1$ here), and $\varepsilon_{\text {eff }}$ is the effective dielectric constant of the substrate. The longitudinal thermal expansion of the substrate will decrease $f$ to first order in $\Delta T$. Although line width does not appear in (5), an increased width will also cause a reduction in $f$ due to an increase in $\varepsilon_{\text {eff }}$. These decreases in $f$ will be offset by increases due to the substrate thickness and also the temperature dependence of the relative permittivity of the substrate $\varepsilon_{r}$, both of which cause reductions in $\varepsilon_{\text {eff }}$. We neglect any contribution from the change in metallisation thickness, which is a second-order effect. We next quantify these different contributions.

The change in the microstrip length $\Delta l$ with temperature is, to first order in changes in temperature [17], [18]

$$
\Delta l=l \alpha_{l} \Delta T
$$

where $\alpha_{l}$ is the linear thermal expansion coefficient. This material property is anisotropic for microwave circuit board (such as the RT/Duroid 5870 used in our experiments) owing to the metal cladding, metals having a much smaller thermal expansion coefficient than plastics. The datasheet quotes $\alpha_{l}$ of 
approximately $+25 \mathrm{ppm} /{ }^{\circ} \mathrm{C}$ for in-plane expansions of the laminate, but $+173 \mathrm{ppm} /{ }^{\circ} \mathrm{C}$ perpendicular to the plane. The increase in length with increasing temperature introduces a small frequency decrease, but more important is the reduction in effective permittivity in (5) caused by the dual effects of increased substrate thickness and temperature-dependent relative permittivity $\varepsilon_{r}$ of the substrate [19]-[21]. Analogous to (6), we may write its change with temperature

$$
\Delta \varepsilon_{r}=\varepsilon_{r} \alpha_{e} \Delta T
$$

where $\alpha_{e}$ is $-115 \mathrm{ppm} /{ }^{\circ} \mathrm{C}$ (for RT/Duroid 5870). The combined effects of increased length, increased substrate thickness and decreased substrate permittivity result in an overall temperature coefficient for the resonant frequency of the microstrip resonator (made from RT/Duroid 5870 laminate) of approximately $+75 \mathrm{ppm} /{ }^{\circ} \mathrm{C}$, i.e., a frequency shift of approximately $+180 \mathrm{kHz} /{ }^{\circ} \mathrm{C}$ for a $2.5 \mathrm{GHz}$ resonator, in broad agreement with experiment (around $+120 \mathrm{kHz} /{ }^{\circ} \mathrm{C}$ ). This temperature variation is a significant systematic measurement error but is eliminated when using our reference resonator.

Finally, we note that an increased temperature will also decrease the $Q$ factor of the empty microstrip, as a result of an increased surface resistance of the copper cladding (as its conductivity has reduced) and increased loss tangent of the substrate. We do not quantify this effect here, but note that it will produce major systematic errors for the measurement of low loss liquids, whose loss actually decreases with increasing temperature, opposite to the trend due to the other materials making up the microstrip. However, we can again account for this systematic error in $Q$ factor (or bandwidth) measurement by the use of our reference resonator.

\section{Use of COMSOL Multiphysics for EM Simulations}

3-D simulations of the fundamental electromagnetic interactions of the liquids with the microwave fields were performed using COMSOL Multiphysics 4.4, including S-parameter calculations. This involves numerical solution of the wave equation in the frequency domain

$$
\nabla \times \mu_{r}^{-1}(\nabla \times \bar{E})-k_{0}^{2}\left(\varepsilon_{r}-\frac{j \sigma}{\omega \varepsilon_{0}}\right) \bar{E}=0
$$

where $\mu_{r}$ is the permeability, $\varepsilon_{r}$ the permittivity, and $\sigma$ the electric conductivity of the materials; $\varepsilon_{0}$ is the permittivity of free space, $k_{0}$ is the wave number in free space, and $\omega$ the angular frequency. The impedance boundary condition is used for the copper surfaces of the resonator, the ground plane, and the inner surfaces of the aluminum box (enclosing the device) in order to consider the copper and aluminum losses. The simulation boundary is the inside surface of the aluminum box. Microwave signals were coupled in and out of the resonator using coaxial (SMA) ports. The complex permittivities of chloroform and water were described in the simulation by using their predicted values from Debye theory. Table I lists the various properties of the materials used in the COMSOL simulations.

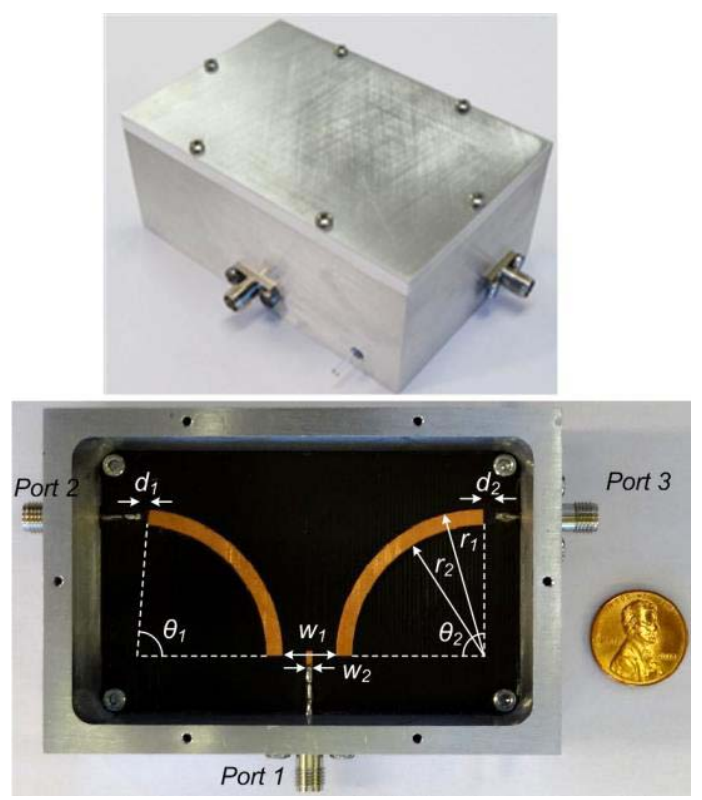

Fig. 3. Photograph of the fabricated sensor with three ports.

TABLE I

Material Properties Used in the Simulation at $25^{\circ} \mathrm{C}$

\begin{tabular}{cccc}
\hline \hline Material & $\varepsilon_{r}$ & & $\sigma(\mathrm{S} / \mathrm{m})$ \\
\hline Air & 1 & 0 \\
Substrate & 2.3 & & $3.3 \times 10^{-4}$ \\
Copper & 1 & & $2.7 \times 10^{7}$ \\
Aluminum & 1 & & $2.0 \times 10^{7}$ \\
Soda glass capillary & 3.8 & & $1.0 \times 10^{-12}$ \\
\hline & $\varepsilon_{s}$ & $\varepsilon_{\infty}$ & $\tau(p \mathrm{~s})$ \\
\hline Chloroform [22] & 4.72 & 2.50 & 7.96 \\
Water [23] & 78.4 & 5.16 & 8.27 \\
\hline \hline
\end{tabular}

\section{LabVIEW Interface}

As has been described, in order to obtain dielectric measurements, accurate measurements of the resonator $Q$ and resonant frequency are required. Instrument noise is minimized by extracting resonator parameters from the coefficients of a fitted Lorentzian curve to measured power transmission coefficient in the frequency domain. The power transmission coefficients between ports 1 and 2, or between 1 and 3, can be written generally as

$$
\left|S_{21,31}\right|^{2}=\frac{4 g^{2}}{(1+2 g)^{2}+4 Q_{o}^{2}\left(\frac{f-f_{0}}{f_{0}}\right)^{2}}=\frac{P_{0}}{1+4 Q_{L}^{2}\left(\frac{f-f_{0}}{f_{0}}\right)^{2}}
$$

where $g$ is the coupling coefficient. The resonators are designed so that this is equal, i.e., symmetric, at each port, $f_{0}$ is the resonant frequency and $P_{\mathrm{o}}$ is the power transmission coefficient at resonance. This sets the relationship between the loaded and unloaded $Q$ as $Q_{L}=Q_{o}\left(1-\sqrt{P}_{o}\right)$. 


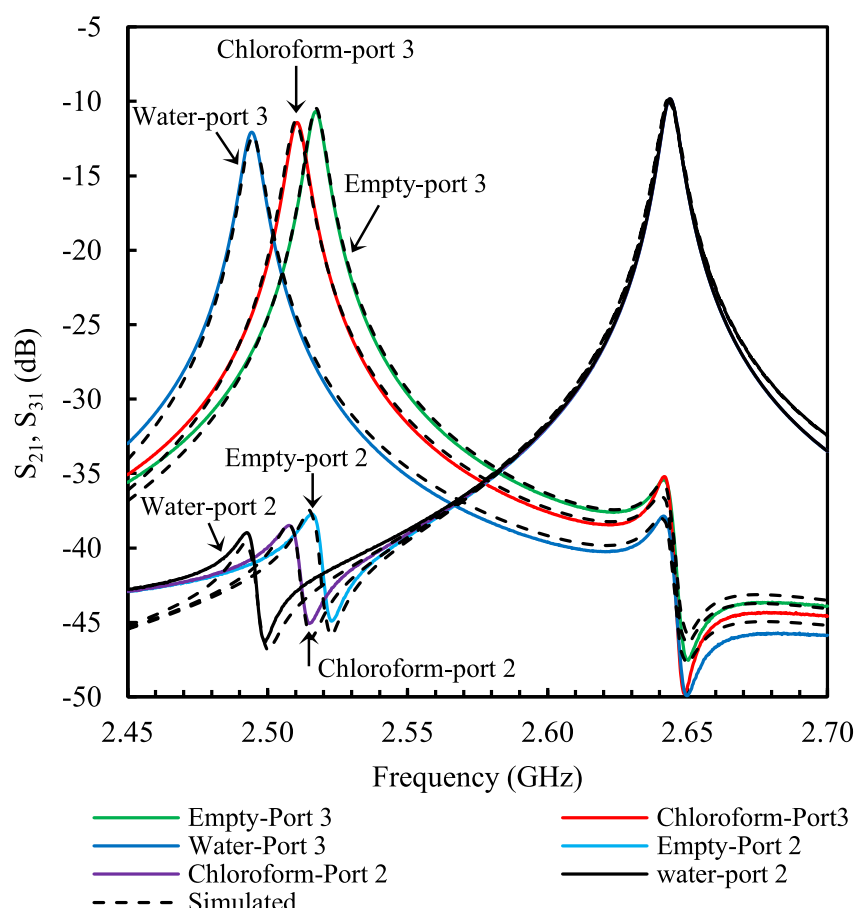

(a)

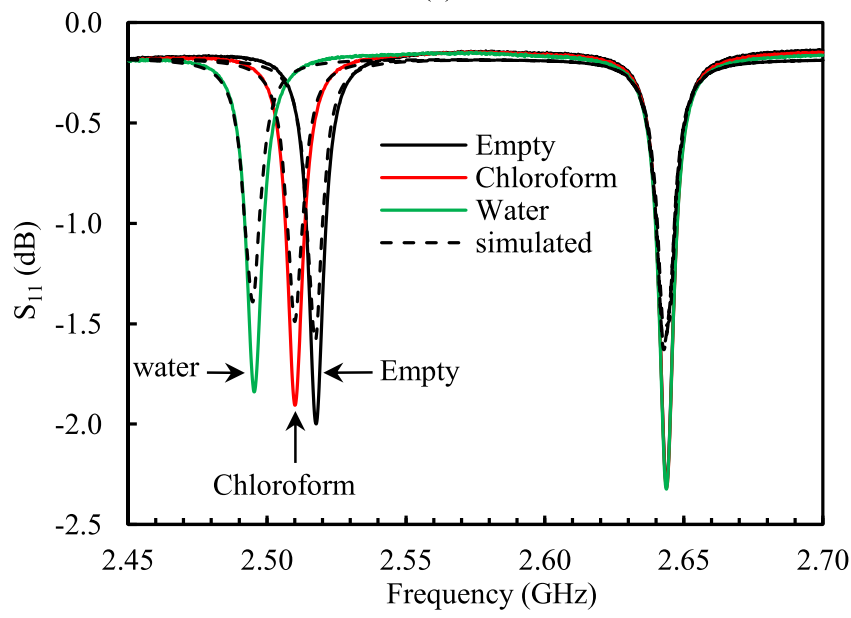

(b)

Fig. 4. Measured and simulated S-parameters of the sensor with empty, chloroform, and water filled capillary. (a) $\left|S_{21}\right|$ and $\left|S_{31}\right|$. (b) $\left|S_{11}\right|$.

Furthermore, this method is often regarded as the most accurate for low SNR, as is the case here with low resonator $Q$, unlike a marker-based bandwidth approach using data taken directly from the network analyzer.

A program was created in LabVIEW to interface with the network analyzer and simultaneously measure $S_{21}$ and $S_{31}$. The curve fitting procedure, also implemented in LabVIEW, utilizes the Levenberg-Marquardt algorithm to find a fit which minimizes the mean squared error between the data and fit.

In an environment where the temperature is changing, it is important that frequency sweep times are minimized in order to reduce the effect of a changing resonant response during the sweep. This requirement necessitates the reduction of the number of frequency points in the sweep, which in turn requires the reduction in measurement span in order to

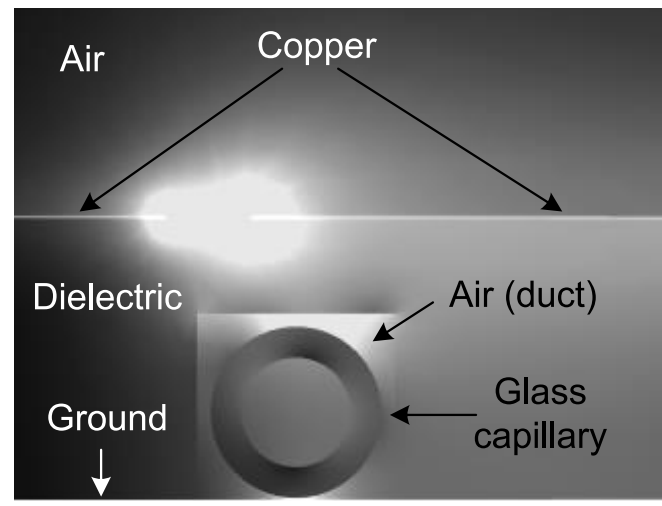

(a)

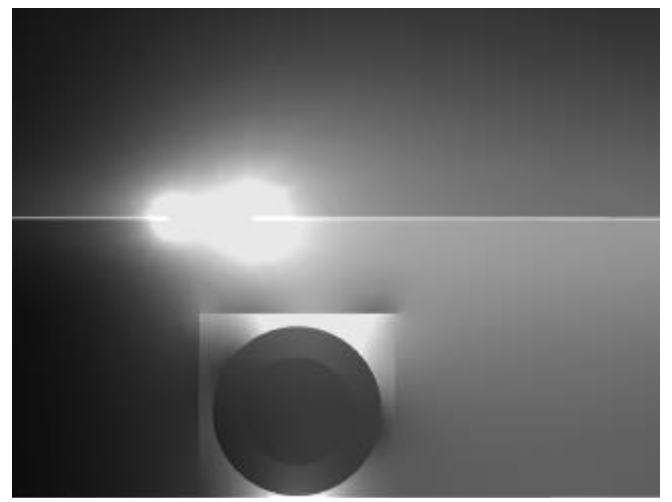

(b)

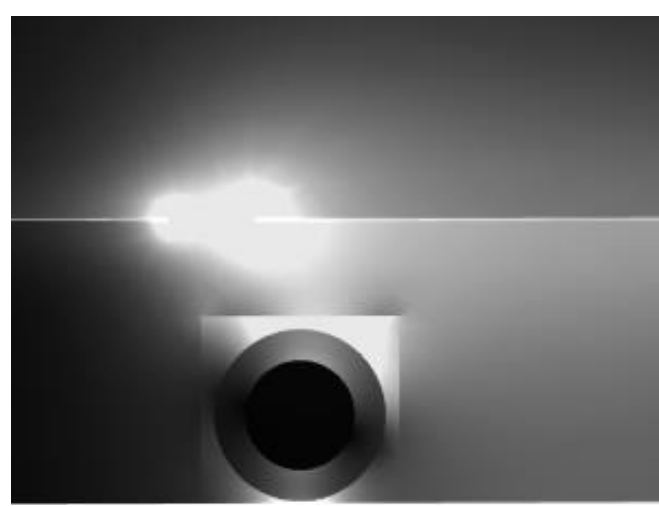

(c)

Fig. 5. Electric field distribution (in $\mathrm{V} / \mathrm{m}$ ) on the cross section of the end of the sensing resonator of the sensor, generated by a COMSOL simulation with (a) empty capillary, (b) chloroform-filled capillary, and (c) water-filled capillary.

maintain accuracy. Therefore, the measurement program was designed to continually adjust the measurement window to track the resonance peak.

\section{DESIGN AND REALIZATION}

The DMS design is based on the idea of two microstrip resonator sensors excited simultaneously (and in parallel) with one input port. The first sensor is used to characterize the liquid sample and the second one is dedicated to determine the change in the ambient temperature, since the liquid and all resonator elements (copper and dielectric dimensions, and material properties) are functions of temperature. A quarter 
TABLE II

Dimensions of THE SENSOR SHOWN IN Fig. 3 (All Dimensions ARE IN mm)

\begin{tabular}{ccccc}
\hline \hline $\mathrm{I}$ & $\mathrm{r}_{\mathrm{i}}$ & $\theta_{\mathrm{i}}$ (deg.) & $\mathrm{w}_{\mathrm{i}}$ & $\mathrm{d}_{\mathrm{i}}$ \\
\hline 1 & 24.9 & 85 & 10.2 & 1.6 \\
2 & 27.9 & 90 & 1.0 & 1.6 \\
\hline \hline
\end{tabular}

ring microstrip line is adopted for the design of the sensor, which is a half wavelength resonator with open circuit ends (at which the electric field will be a maximum). The effective permittivity $\varepsilon_{\text {eff }}$ can be calculated used standard means (e.g., LineCalc, within Keysight's ADS software). The resonators were mechanically milled from an RT/Duroid 5870 laminate (Rogers Corporation), with a dielectric layer of nominal thickness of $3.18 \mathrm{~mm}$, relative permittivity of $2.33 \pm 0.02$, loss tangent of 0.0012 , and a copper cladding of thickness $35 \mu \mathrm{m}$. All of the milled resonator dimensions are listed in Table II.

In order to avoid undesirable field coupling between the two resonators [24], they were designed to have two different resonant frequencies $(2.53 \mathrm{GHz}$ for the liquid loaded resonator, with the reference resonator at $2.65 \mathrm{GHz}$, i.e., $120 \mathrm{MHz}$ difference between them). This was done by making them slightly different lengths, as shown in Fig. 3 and Table II. This minimizes any interference between them when liquid sample is under test, and brings the frequency of the loaded resonator into the range 2.45 to $2.50 \mathrm{GHz}$, i.e., one of the industrially important IMS bands. The coupling gap $d$ of $1.6 \mathrm{~mm}$ was chosen for a $-10 \mathrm{~dB}$ insertion loss at resonance, providing a large signal to noise ratio for $S_{21}$ measurements, without excessive loading of the cavity due to strong coupling.

The position of the capillary was chosen to be between the end of the microstrip line resonator and the ground through the dielectric, as shown in Fig. 2, to obtain maximum perturbation of the resonator as the electric field will be highest there. A duct of dimensions $2 \mathrm{~mm} \times 2 \mathrm{~mm} \times 50 \mathrm{~mm}$ was milled on the back side of the microstrip board to hold the capillary and then the duct was finally layered by copper sheet. A soda glass capillary (SAMCO) with inner radius of $0.7 \mathrm{~mm}$, outer radius of $1 \mathrm{~mm}$, and relative permittivity of 3.8 is used to hold the liquid.

We note that several approaches have been used elsewhere to increase the $Q$ factors of the resonators, which helps to increase the sensitivity of the sensor, all of which can be applied to our sensor. Very effective is the generation of negative resistance [25] in a passive ring resonator enabled by an active feedback loop, which compensates for the loss of the resonator and hence increases its quality factor greatly.

In our work, the microstrip resonator board was set inside a rectangular cavity (an aluminum box), which increases the quality factor from 80 to 360 by reducing the radiation loss from the structure. This is close to the optimal $Q$ expected for the resonators, given the loss tangent of the substrate and the surface resistance of the copper conductors, each of which give a dielectric and conductor $Q$ of about 800 each, resulting in an overall $Q$ of around 400 . The size of the cavity was designed

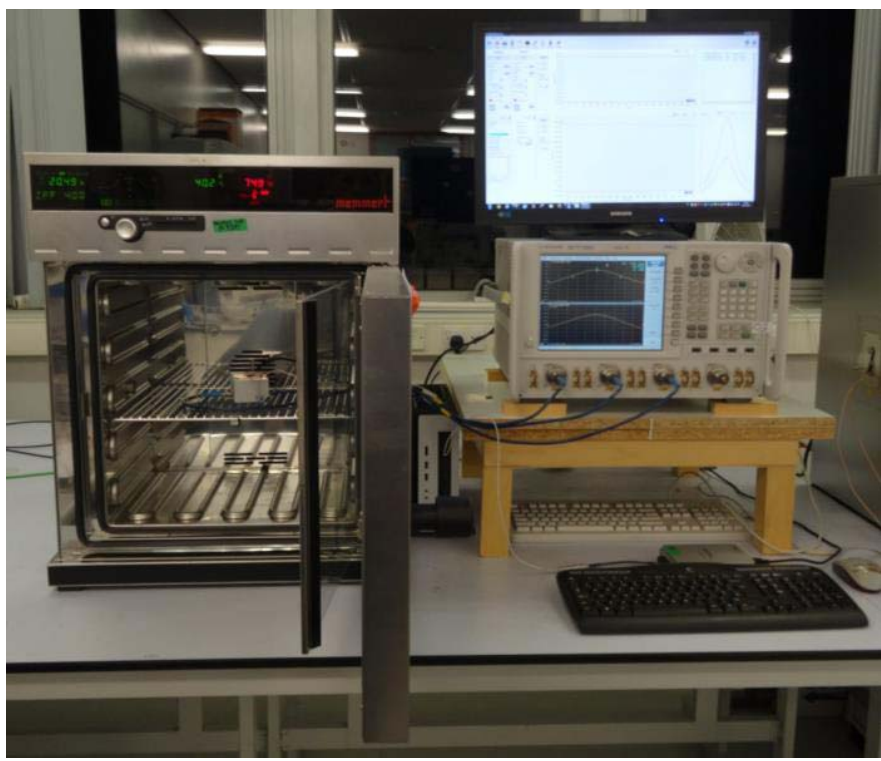

Fig. 6. Photograph the bench-top experimental assembly. The Sensor is put inside a computer-controlled oven and connected to an Agilent PNA-L N5232A network analyzer under LabVIEW program control.

to be small enough so that its own resonant frequencies were much larger than the resonant frequencies of the sensor to avoid the interference between them. The microstrip board was fixed on the bottom of the box by four metal screws, as shown in Fig. 2, and the capillary was inserted into the microstrip board through the holes in the outer rectangular cavity. SMA connectors were connected to the sensor via the box to connect to the network analyzer. COMSOL Multiphysics simulations were performed for the sensor with empty, chloroform filled, and water filled capillaries at nominally $25^{\circ} \mathrm{C}$.

The complex permittivities of the liquids (water and chloroform) are assumed to have real and imaginary parts which are well described by Debye theory [15]. Values of the Debye parameters for water and chloroform at an ambient temperature of $25^{\circ} \mathrm{C}$ were taken from [22] and [23]. The simulated and measured results are illustrated in Fig. 4, showing excellent agreement between the two.

The distribution of the electric field on the cross section of the coupling end of the sensing resonator is illustrated in Fig. 5, which was performed by using COMSOL Multiphysics. Fig. 5 shows three cases: empty capillary, chloroformfilled capillary, and water-filled capillary. In the case of the empty capillary, the electric field concentrates between the edges of the resonator and coupling strips, and the top of the capillary when excited at the $2.517 \mathrm{GHz}$ resonant frequency. The presence of the chloroform in the capillary at its resonant frequency of $2.510 \mathrm{GHz}$, as shown in Fig. 5(b), increases the intensity of the electric field at the polar regions outside of the capillary. For a water-filled capillary at its resonant frequency of $2.494 \mathrm{GHz}$, the electric field is strongly concentrated between the edges of the resonator and the capillary, much more than in the two previous cases. The electric field magnitude inside the capillary is much less than that outside, due to the highly polar nature of water, causing a large reduction (depolarisation) of the internal field. 


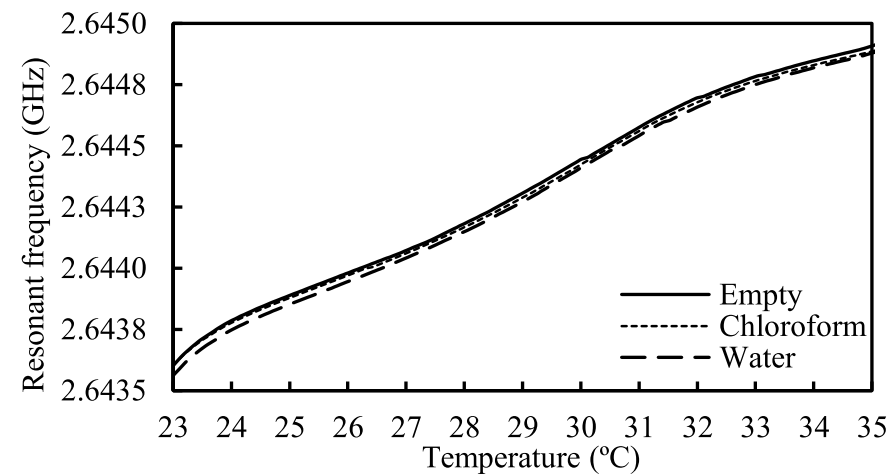

(a)

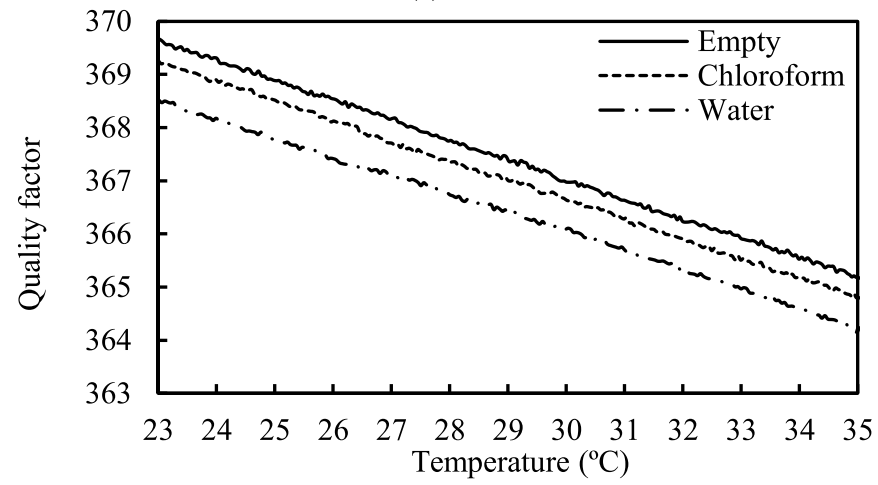

(b)

Fig. 7. Port 2 measurements of the reference resonator with temperature, when the sensor resonator contains empty, chloroform, and water filled capillaries. (a) Resonant frequency. (b) Quality factor.

\section{RESUlts AND Discussion}

To verify the sensor performance a temperature scan was applied. A computer-controlled oven with fine temperature control to $\pm 0.1{ }^{\circ} \mathrm{C}$ (Memmert, IPP 400) was used to heat the sensor from $23{ }^{\circ} \mathrm{C}$ to $35{ }^{\circ} \mathrm{C}$. The rise time was set to be very long $(5 \mathrm{~h})$ to ensure approximate thermal equilibrium among all parts of the sensor, capillary, and the liquid. The benchtop assembly of the sensor inside the oven, with network analyzer with control computer (running the LabVIEW control program) is shown in Fig. 6. As the sensor has three ports (one for input and two for outputs), a four port PNA-L N5232A network analyzer (Agilent technologies) was used to measure the S-parameters $\left(S_{11}, S_{21}, S_{31}\right)$ of the sensor as functions of temperature. Microwave power levels were $-10 \mathrm{dBm}$, so there was negligible microwave heating of the capillary contents. The LabVIEW program also collects temperature measurements from a platinum resistance thermometer mounted, on the microstrip ground plane by means of a small fixing screw.

As shown in Fig. 7(a), when the microwave measurement was taken from port 2 (the reference port) when undertaking the temperature sweep, the resonant frequency was found to increase with temperature. This behaviour is explained in Section II-B, as the effect of the thermal expansion of the substrate thickness with temperature is dominant over all other parameters (copper thermal expansion and the temperature coefficient of the dielectric constant of the substrate). Moreover, the microwave losses increase with increasing temperature, as shown in Fig. 7(b). This is due to first-order increases

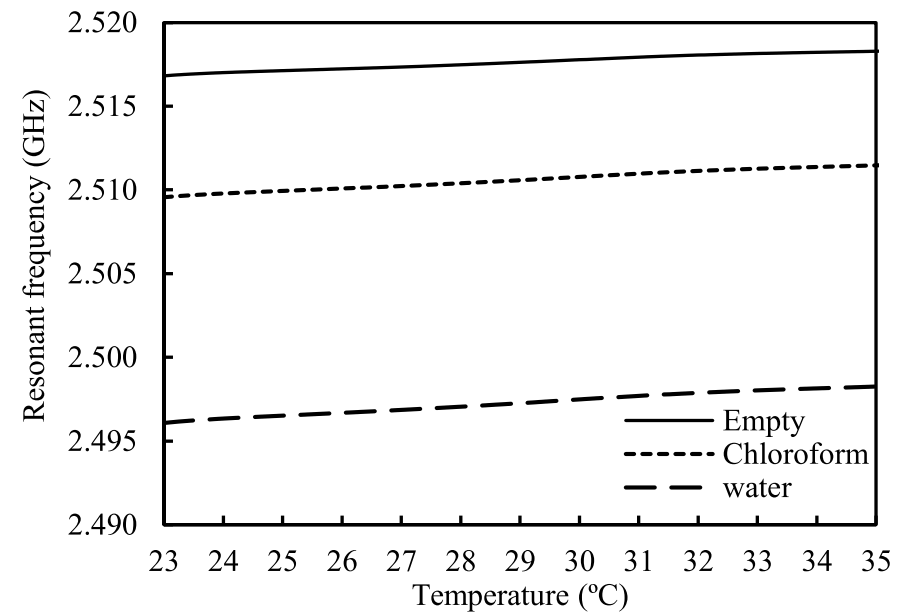

(a)

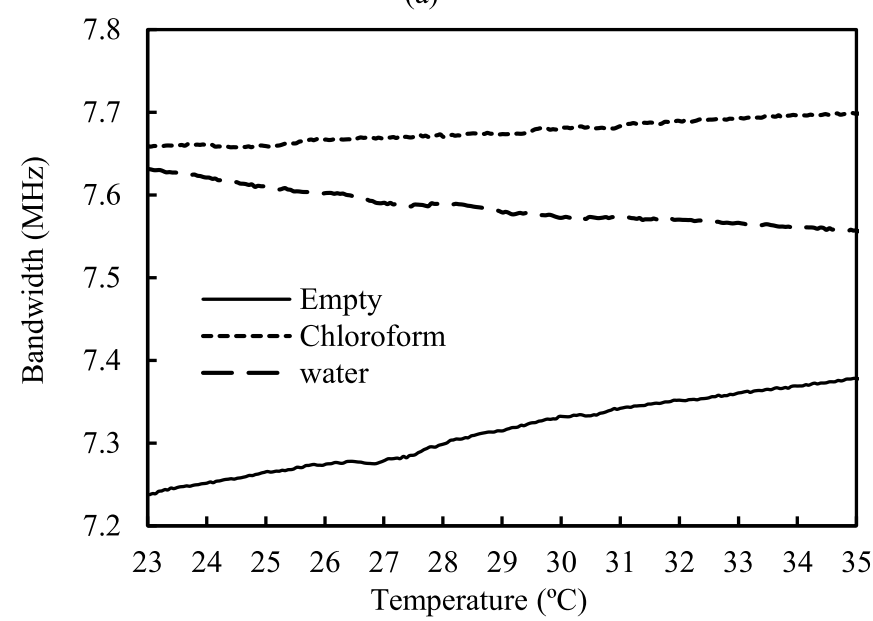

(b)

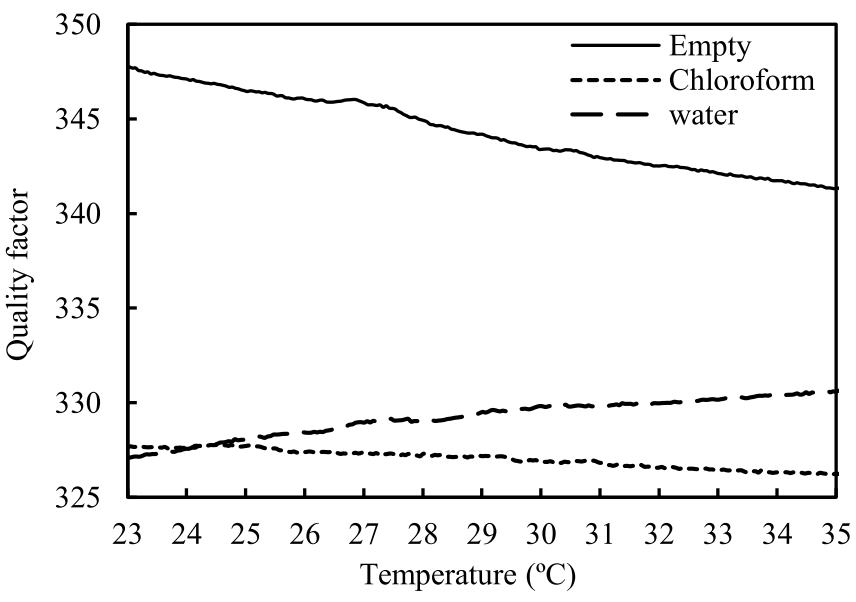

(c)

Fig. 8. Port $3 S_{21}$ measurements with temperature for empty, chloroform, and water sample. (a) Resonant frequency. (b) Bandwidth. (c) Quality factor.

in the surface resistance of the copper and loss tangent of the substrate, in addition to the changes in dielectric property of the liquid, more details of Fig. 7(b) are illustrated at the end of this section.

The overall loss (i.e., bandwidth) of the water-loaded microstrip decreases with increasing temperature since the water dielectric loss dominates, and from the Debye model this decreases with increasing temperature. Chloroform, on the 


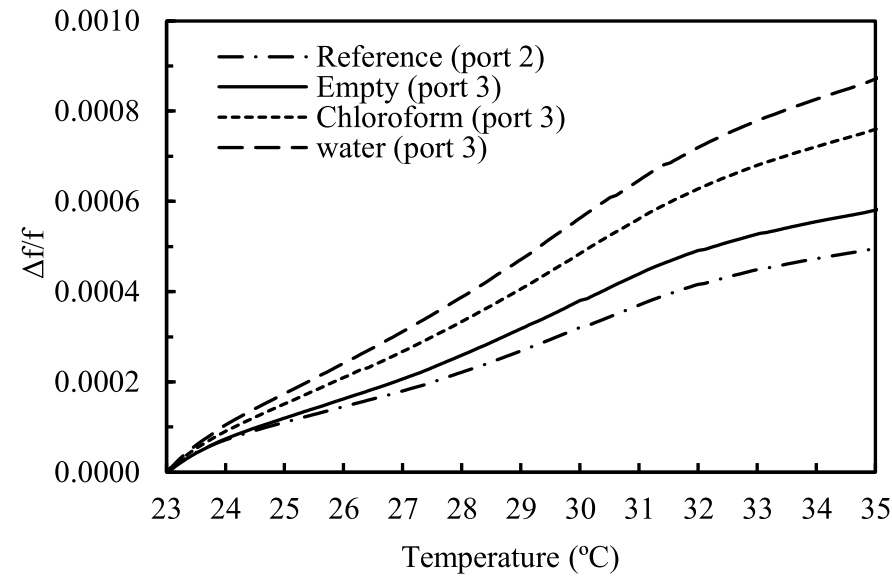

(a)

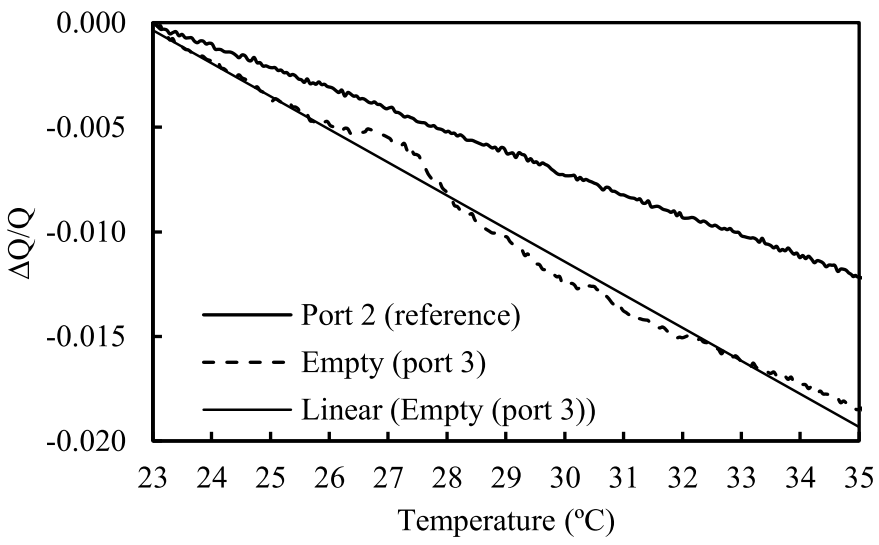

(b)

Fig. 9. Change in the fractional resonant frequency with temperature (a) $\Delta$ f/f of both resonators (ports 2 and 3) and (b) $\Delta$ Q/Q of both resonators (ports 2 and 3 ).

other hand, is much less lossy than water, so in this case the microstrip losses dominate, thus explaining the opposite trends for the two liquids observed in Fig. 8.

Comparatively, the changes in resonant frequency measured from port 3 when the capillary was empty were very similar to those from port 2 (for the sensor resonator), with some minor differences as the structures are not identical, e.g., due to the present of the duct in one, as shown in Fig. 7(a). The resonant frequency of the reference resonator (port 2) at $23{ }^{\circ} \mathrm{C}$ was $2.6436 \mathrm{GHz}$, and at $35^{\circ} \mathrm{C}$ was $2.6449 \mathrm{GHz}$, meaning that the shift in the resonant frequency was $+1.3 \mathrm{MHz}$. In the case of the empty capillary resonator (port 3 ), the resonant frequencies at $23{ }^{\circ} \mathrm{C}$ and $35^{\circ} \mathrm{C}$ were 2.5168 and $2.5183 \mathrm{GHz}$, respectively, and the shift in this case was slightly higher $(+1.5 \mathrm{MHz})$.

The sensor then was tested with two liquids: chloroform (weakly polar) and water (strongly polar). The change in the resonant frequency, $3 \mathrm{~dB}$ bandwidth, and quality factor with temperature from port 3 with a chloroform filled capillary is shown in Fig. 8. The shift in resonant frequency over the range of temperature sweep is about $+1.91 \mathrm{MHz}$. There are also changes in the bandwidth and quality factor. In the case of water, the shifts in resonant frequency, losses, bandwidth, and quality factor will be larger, as illustrated in Fig. 8. The resonant frequency increased from $2.4961 \mathrm{GHz}$ at $23{ }^{\circ} \mathrm{C}$ to 2.4983 at $35{ }^{\circ} \mathrm{C}$, a shift of $+2.2 \mathrm{MHz}$.

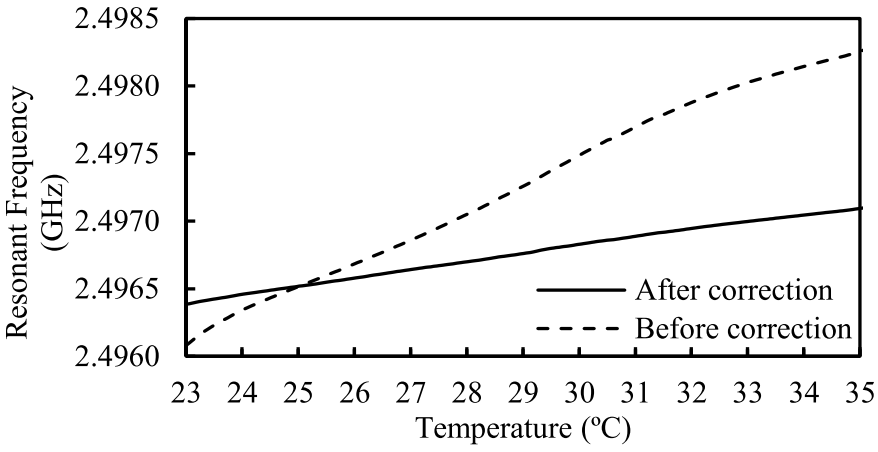

(a)

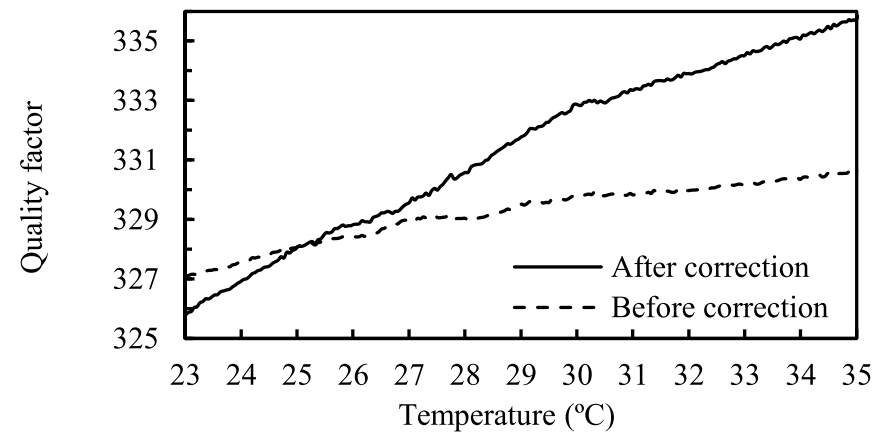

(b)

Fig. 10. Port 3 corrected measurements with temperature for the water sample. (a) Resonant frequency. (b) Quality factor.

The resulting fractional changes in resonant frequencies of both resonators (ports 2 and 3) with temperature are shown in Fig. 9(a). Moreover, Fig. 9(b) shows the change in the fractional quality factor with temperature between the reference resonator (port 2) and that of an empty capillary (port 3 ). The results can be approximated by a linear relationship, useful for the temperature correction.

All these results demonstrate the need to know precisely the effect of instantaneous temperature on both the liquid permittivity and all other materials, in order to calibrate the measurements. The reference channel (port 2) can then provide the information about temperature which can be used to obtain accurate, corrected values of the liquid permittivity.

The temperature correction of the results proceeds by removing the changes in the resonant frequency and quality factor arising from the changes of temperature on all parts of the resonator except the liquid sample. The changes in resonant frequency and quality factor for all parts of the resonator (except the liquid) can be found from the measurements at port 2 (the reference resonator). The key point here is that the contribution to the fractional frequency shift $\Delta f / f$ due to temperature changes is the same for both resonators and so can be deduced from measurement of the reference resonator. As shown in Fig. 9(a), the approximate relationship between $\Delta f / f$ of the reference resonator (port 2) and $\Delta f / f$ of the empty capillary (port 3 ) can be easily found as

$$
\Delta f / f_{\text {empty }(\text { port } 3)}=K_{f} \bullet \Delta f / f_{\text {ref(port2) }}
$$

where $K_{f}$ is constant which can be calculated from Fig. 9(a) $\left(K_{f}=1.15\right)$. Similarly, as shown in Fig. 9(b), the change in the quality factor $\Delta Q$ (or equivalently, changes in $3 \mathrm{~dB}$ 


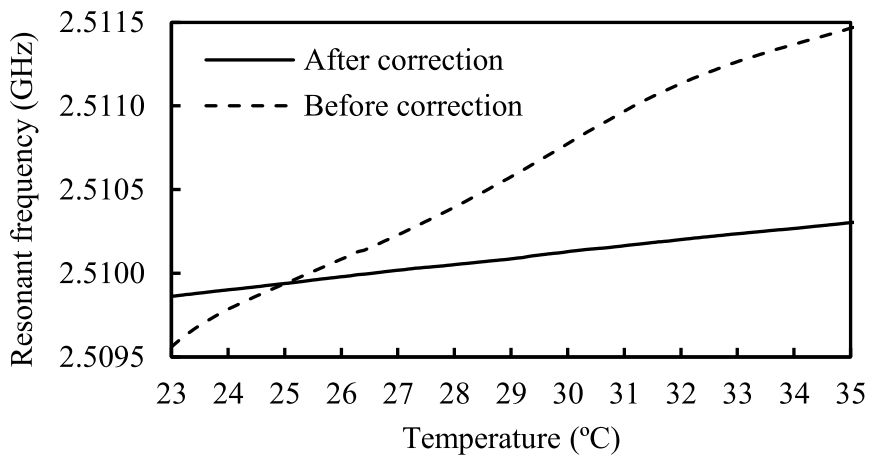

(a)

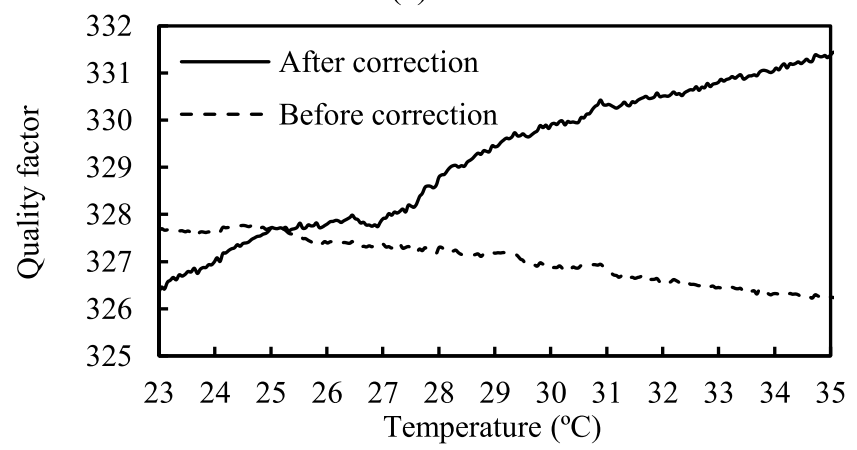

(b)

Fig. 11. Port 3 corrected measurements with temperature for the chloroform sample. (a) Resonant frequency. (b) Quality factor.

bandwidth, since changes in $Q$ are small) of the reference resonator (port 2) and that for the empty capillary (port 3) can be linked by

$$
\Delta Q / Q_{\text {empty(port3) }}=K_{Q} \bullet \Delta Q / Q_{\text {ref(port2) }} .
$$

In this case $K_{Q}=1.60$. From (10) and (11), the resonant frequency and the quality factor in the case of the empty capillary of port 3 can be found instantaneously from the measurements of port 2 as function of temperature. To demonstrate how temperature drifts can result in large errors in extraction of complex permittivity, we assume room temperature $\left(25^{\circ} \mathrm{C}\right)$ as the reference temperature to calibrate our results. Then the changes in the resonant frequency and quality factor of the empty capillary as function of temperature of port 3 can be found as

$$
\begin{aligned}
\Delta f(T) & =f(T)-f\left(25^{\circ}\right) \\
\Delta Q(T) & =Q(T)-Q\left(25^{\circ}\right) .
\end{aligned}
$$

The corrected values of the resonant frequency and the quality factor (for water or chloroform) will be the instantaneous measurements minus the values of $\Delta f(T)$ and $\Delta Q(T)$ of the empty capillary, respectively, using

$$
\begin{aligned}
f_{\text {corrected }} & =f_{\text {measured }}-\Delta f(T) \\
Q_{\text {corrected }} & =Q_{\text {measured }}-\Delta Q(T) .
\end{aligned}
$$

The corrected results (resonant frequency and quality factor) for water and chloroform are shown in Figs. 10 and 11, respectively, where the effect of temperature on the resonator parts (except the liquid sample) was removed. An optimization

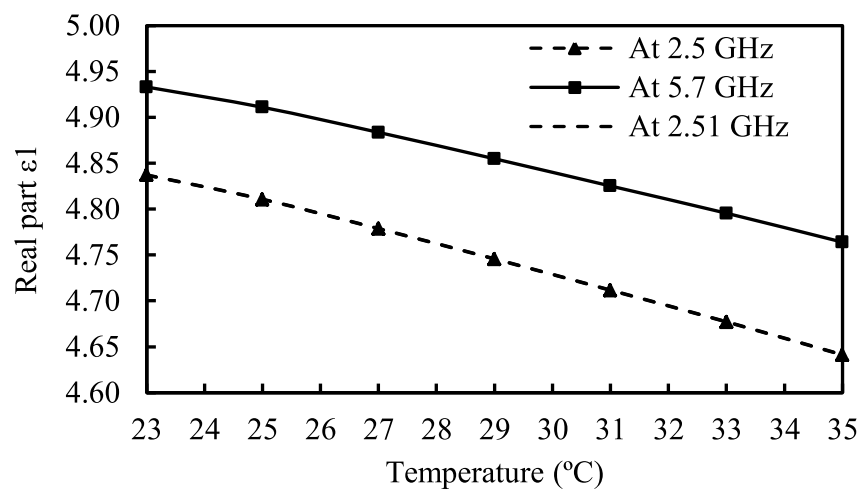

(a)

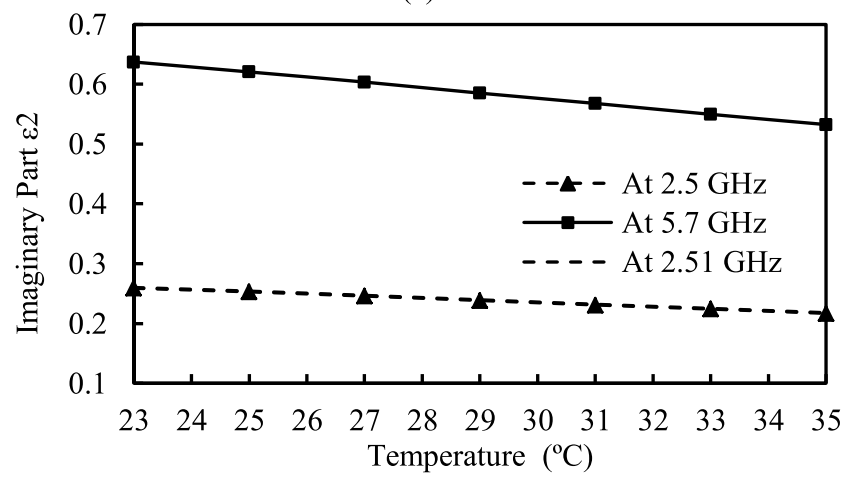

(b)

Fig. 12. Temperature dependence of the complex permittivity of chloroform measured using a cylindrical cavity at 2.5 and $5.7 \mathrm{GHz}$. (a) Real part. (b) Imaginary part.

routine is used to calculate the complex permittivity of the samples, which is based on the matching of the simulated and experimental results [4], [5], [10]. In this, rather than using the approximate perturbation analysis, we use the full COMSOL model to link the complex permittivity with changes in the resonator parameters, thus accounting explicitly for reconfiguration of electric fields around the sample as the permittivity changes. In the case of water, the values of its complex permittivity as a function of temperature have been calculated from [26] and used as reference for comparison with the measured and corrected results.

The random errors of $\pm 0.1 \%$ for permittivity $\varepsilon$ in Table III arise as a result of the random errors in the resonator parameters (also shown in Table III), estimated from repeated measurements of the same sample within the same capillary. The differences of the magnitude of the complex permittivity values $|\varepsilon|$, which are calculated from the measured (uncorrected) results, vary from $2.6 \%$ to $11.4 \%$ compared to the expected values, depending on the temperature. In the case of the corrected results, these differences reduce to around $0.5 \%$, as illustrated in Table III. The same procedure was repeated for the chloroform sample.

There are no reliable, published data for the complex permittivity of chloroform as a function of temperature and frequency. For this reason, it was measured as a function of temperature in separate perturbation experiments using a cylindrical microwave cavity [12], [27]. The complex permittivity of chloroform was measured over the 
TABLE III

Comparison Between Calculated and Measured Values of Solvents Complex Permittivity Before and After CORRECTION OF WATER AND RELATIVE ERROR

\begin{tabular}{cccccccccc}
\hline \hline \multirow{2}{*}{$\left({ }^{\circ} \mathrm{C}\right)$} & $\begin{array}{c}\mathrm{f}(\mathrm{GHz}) \\
\text { measured } \\
\pm 0.00001\end{array}$ & $\begin{array}{c}\mathrm{f}(\mathrm{GHz}) \\
\text { corrected } \\
\pm 0.00001\end{array}$ & $\begin{array}{c}\mathrm{Q} \\
\text { measured } \\
\pm 0.1\end{array}$ & $\begin{array}{c}\mathrm{Q} \\
\text { corrected } \\
\pm 0.1\end{array}$ & $\begin{array}{c}\varepsilon \text { calculated } \\
{[26]}\end{array}$ & $\begin{array}{c}\varepsilon \text { measured } \\
\pm 0.1 \%\end{array}$ & $\begin{array}{c}\text { diff } \\
\text { in }|\varepsilon|\end{array}$ & $\begin{array}{c}\varepsilon \text { corrected } \\
\text { diff } \\
\text { in }|\varepsilon|\end{array}$ \\
\hline 23 & 2.49608 & 2.49639 & 327.1 & 325.8 & $77.74-j 9.88$ & $81.70-j 9.80$ & $4.9 \%$ & $78.50-j 10.00$ & $0.9 \%$ \\
25 & 2.49652 & 2.49652 & 328.1 & 328.1 & $77.15-j 9.33$ & $77.15-j 9.33$ & $0.0 \%$ & $77.15-j 9.33$ & $0.0 \%$ \\
27 & 2.49686 & 2.49664 & 328.0 & 329.6 & $76.54-j 8.81$ & $74.50-j 9.00$ & $2.6 \%$ & $76.30-j 8.80$ & $0.3 \%$ \\
29 & 2.49726 & 2.49676 & 329.5 & 331.8 & $75.94-j 8.30$ & $72.00-\mathrm{j} 8.60$ & $5.1 \%$ & $75.50-\mathrm{j} 8.20$ & $0.5 \%$ \\
31 & 2.49770 & 2.49689 & 329.8 & 333.3 & $75.34-j 7.85$ & $70.00-\mathrm{j} 8.15$ & $7.0 \%$ & $75.00-\mathrm{j} 7.80$ & $0.5 \%$ \\
33 & 2.49802 & 2.49700 & 330.2 & 334.5 & $74.75-j 7.45$ & $68.00-\mathrm{j} 7.80$ & $8.9 \%$ & $74.40-\mathrm{j} 7.40$ & $0.5 \%$ \\
35 & 2.49826 & 2.49709 & 330.6 & 335.8 & $74.15-j 7.05$ & $65.50-j 7.50$ & $11.4 \%$ & $73.80-j 7.00$ & $0.5 \%$ \\
\hline \hline
\end{tabular}

TABLE IV

Comparison Between Calculated and Measured Values of Solvents Complex Permittivity Before and After CORRECTION OF CHLOROFORM AND RELATIVE ERROR

\begin{tabular}{cccccccccc}
\hline \hline \multirow{2}{*}{$\left({ }^{\circ} \mathrm{C}\right)$} & $\begin{array}{c}\mathrm{f}(\mathrm{GHz}) \\
\text { measured } \\
\pm 0.00001\end{array}$ & $\begin{array}{c}\mathrm{f}(\mathrm{GHz}) \\
\text { corrected } \\
\pm 0.00001\end{array}$ & $\begin{array}{c}\text { Q } \\
\text { measured } \\
\pm 0.1\end{array}$ & $\begin{array}{c}\text { corrected } \\
\pm 0.1\end{array}$ & $\begin{array}{c}\varepsilon \text { measured } \\
\text { (cavity) }\end{array}$ & $\begin{array}{c}\varepsilon \text { measured } \\
\pm 0.1 \%\end{array}$ & $\begin{array}{c}\text { diff } \\
\text { in }|\varepsilon|\end{array}$ & $\begin{array}{c}\varepsilon(f, T) \\
\text { corrected }\end{array}$ & $\begin{array}{c}\text { diff } \\
\text { in }|\varepsilon|\end{array}$ \\
\hline 23 & 2.50956 & 2.50986 & 327.6 & 326.3 & $4.84-j 0.26$ & $5.00-j 0.25$ & $3.3 \%$ & $4.83-j 0.27$ & $0.2 \%$ \\
25 & 2.50994 & 2.50994 & 327.7 & 327.7 & $4.81-\mathrm{j} 0.25$ & $4.81-\mathrm{j} 0.25$ & $0.0 \%$ & $4.81-\mathrm{j} 0.25$ & $0.0 \%$ \\
27 & 2.51023 & 2.51002 & 327.4 & 327.9 & $4.78-\mathrm{j} 0.25$ & $4.63-\mathrm{j} 0.26$ & $3.1 \%$ & $4.77-\mathrm{j} 0.26$ & $0.2 \%$ \\
29 & 2.51058 & 2.51009 & 327.2 & 329.4 & $4.75-\mathrm{j} 0.24$ & $4.60-\mathrm{j} 0.27$ & $3.1 \%$ & $4.74-\mathrm{j} 0.25$ & $0.2 \%$ \\
31 & 2.51097 & 2.51016 & 326.8 & 330.3 & $4.71-\mathrm{j} 0.23$ & $4.55-\mathrm{j} 0.27$ & $3.3 \%$ & $4.69-\mathrm{j} 0.24$ & $0.4 \%$ \\
33 & 2.51126 & 2.51024 & 326.5 & 330.8 & $4.68-\mathrm{j} 0.22$ & $4.40-\mathrm{j} 0.27$ & $5.9 \%$ & $4.67-\mathrm{j} 0.23$ & $0.2 \%$ \\
35 & 2.51147 & 2.51030 & 326.2 & 331.4 & $4.64-\mathrm{j} 0.22$ & $4.24-\mathrm{j} 0.28$ & $8.5 \%$ & $4.63-\mathrm{j} 0.22$ & $0.2 \%$ \\
\hline \hline
\end{tabular}

temperature range $23{ }^{\circ} \mathrm{C}-35{ }^{\circ} \mathrm{C}$ using the two resonant frequencies of the $\mathrm{TM}_{010}$ and $\mathrm{TM}_{020}$ modes of the cylindrical cavity (at 2.5 and $5.7 \mathrm{GHz}$, respectively) placed within the oven. The former resonant frequency is very close to the DMS resonant frequency of $2.51 \mathrm{GHz}$. The temperature-dependent complex permittivity data for chloroform are plotted as a function of temperature in Fig. 12. The error for chloroform reduces from the range of $3.1 \%-8.5 \%$ for the uncorrected results, to around $0.3 \%$ for the corrected results. The corresponding calculations of the complex permittivity of water and chloroform before and after the correction are illustrated in Tables III and IV, respectively.

However, due to the interaction between the two channels (the reference and sensing channel) there is some variation in the resonant frequency and the quality factor of the reference channel (port 2) owing to the presence of the liquid in the sample resonator at the same temperature, as shown in Fig. 7. This design of the two resonators with the common input is similar to a duplexer structure, where some EM interaction between the two channels is inevitable [24], [28]. The variation in the reference frequency due to the presence of different liquids at $25^{\circ} \mathrm{C}$ is $20 \mathrm{KHz}$ in the case of the chloroform and $40 \mathrm{kHz}$ in case of water. These small shifts in the resonant frequency and the quality factor of the reference channel may produce some error in the extracting the relative permittivity of the liquid sample. To solve this problem, and to make the measurement even more accurate, two modifications can be added. First, the feed method of the two channels can be changed to a T-junction in order to increase the distance between them and consequently decrease the interaction between their electromagnetic fields. Second, the difference between the resonant frequencies of the two resonators can be increased to reduce the interaction. These options will be investigated in the next generation of the DMS sensor.

\section{CONClusion}

This paper proposes a new type of microwave microfluidic sensor with two modes, based on a half wavelength microstrip resonator. One of these is used to measure the dielectric properties of a liquid, while the other is used to correct for any changes in ambient temperature. Such temperature changes affect both the dielectric properties of the liquid and the geometrical and electrical properties of the host resonator, so they have to carefully decoupled. This is what our dual mode resonator provides. A temperature sweep from $23{ }^{\circ} \mathrm{C}$ to $35{ }^{\circ} \mathrm{C}$ was conducted to verify the sensor performance. Two polar 
liquids (water and chloroform) were tested using the sensor with varying temperature to show the effects of temperature on the measurements and how these can be calibrated using the reference resonator. It is clear that when measuring lower loss liquids such as chloroform, a correction procedure like that permitted by our sensor is absolutely necessary for reliable permittivity measurement. Even when measuring water, where the dielectric properties of the liquid dominate the temperature dependence, systematic errors of more than $10 \%$ will be introduced when ignoring the effects of the host resonator. Finally, we note that our sensor can be redesigned to measure the permittivity of two liquids or more by adding more microstrip branches, which opens up the ability to monitor simultaneously the permittivity of several liquids with temperature.

\section{REFERENCES}

[1] T. Chretiennot, D. Dubuc, and K. Grenier, "A microwave and microfluidic planar resonator for efficient and accurate complex permittivity characterization of aqueous solutions," IEEE Trans. Microw. Theory Techn., vol. 61, no. 2, pp. 972-978, Feb. 2013.

[2] A. Ebrahimi, W. Withayachumnankul, S. Al-Sarawi, and D. Abbott, "High-sensitivity metamaterial-inspired sensor for microfluidic dielectric characterization," IEEE Sensors J., vol. 14, no. 5, pp. 1345-1351, May 2014.

[3] D. J. Rowe, S. Al-Malki, A. A. Abduljabar, A. Porch, D. A. Barrow, and C. J. Allender, "Improved split-ring resonator for microfluidic sensing," IEEE Trans. Microw. Theory Techn., vol. 62, no. 3, pp. 689-699, Mar. 2014.

[4] A. A. Abduljabar, D. J. Rowe, A. Porch, and D. A. Barrow, "Novel microwave microfluidic sensor using a microstrip split-ring resonator," IEEE Trans. Microw. Theory Techn., vol. 62, no. 3, pp. 679-688, Mar. 2014.

[5] A. A. Abduljabar, A. Porch, and D. A. Barrow, "Real-time measurements of size, speed, and dielectric property of liquid segments using a microwave microfluidic sensor," in IEEE MTT-S Int. Microw. Symp. Dig., Tampa, FL, USA, Jun. 2014, pp. 1-4.

[6] Y. Cui, Y. He, and P. Wang, "A quadrature-based tunable radio-frequency sensor for the detection and analysis of aqueous solutions," IEEE Microw. Wireless Compon. Lett., vol. 24, no. 7, pp. 490-492, Jul. 2014.

[7] U. Schwerthoeffer, R. Weigel, and D. Kissinger, "Microwave sensor for precise permittivity characterization of liquids used for aqueous glucose detection in medical applications," in Proc. German Microw. Conf. (GeMiC), Aachen, Germany, Mar. 2014, pp. 1-2.

[8] D. J. Rowe, A. Porch, D. A. Barrow, and C. J. Allender, "Microfluidic microwave sensor for simultaneous dielectric and magnetic characterization," IEEE Trans. Microw. Theory Techn., vol. 61, no. 1, pp. 234-243, Jan. 2013.

[9] V. Pohl, D. Fricke, and A. Mühlbauer, "Correction procedures for the measurement of permittivities with the cavity perturbation method," J. Microw. Power Electromagn. Energy, vol. 30, no. 1, pp. 10-26, 1995.

[10] A. Abduljabar, X. Yang, D. Barrow, and A. Porch, "Microstrip split ring resonator for microsphere detection and characterization," in IEEE MTT-S Int. Microw. Symp. Dig., Phoenix, AR, USA, May 2015, pp. 1-4.

[11] A. A. Abduljabar, X. Yang, D. A. Barrow, and A. Porch, "Modelling and measurements of the microwave dielectric properties of microspheres," IEEE Trans. Microw. Theory Techn., vol. 63, no. 12, pp. 4492-4500, Dec. 2015.

[12] D. M. Pozar, Microwave Engineering. New York, NY, UAS: Wiley, 2005.

[13] A. Khayari, M. Medrano, E. Verlage, M. C. Velázquez-Ahumada, M. J. Freire, and A. Ramos, "Microwave-induced water flow in a microchannel built on a coplanar waveguide," J. Appl. Phys., vol. 110, no. 6, pp. 064912-1-064912-9, Sep. 2011.

[14] R. Somaraju and J. Trumpf, "Frequency, temperature and salinity variation of the permittivity of seawater," IEEE Trans. Antennas Propag., vol. 54, no. 11, pp. 3441-3448, Nov. 2006.

[15] H. Frohlich, Theory of Dielectrics. New York, NY, USA: Oxford Univ. Press, 1958.

[16] S. Ramo, J. R. Whinnery, and T. V. Duzer, Fields and Waves in Communication Electronics. Hoboken, NJ, USA: Wiley, 1994.
[17] T. Ashour, L. Fan, Z. Ding, and K. Chang, "Temperature stability for microstrip delay lines on high permittivity substrates," in IEEE MTT-S Int. Microw. Symp. Dig., vol. 1. San Francisco, CA, USA, Jun. 1996, pp. 95-98.

[18] R. K. Hoffman, Handbook of Microwave Integrated Circuits. Norwood, MA, USA: Artech House, 1987.

[19] F. Mernyei and F. Volgyi, "Simple methods for testing the temperature dependence of microstrip antenna array," in Proc. 20th Eur. Microw. Conf., vol. 1. Budapest, Hungary, 1990, pp. 365-370.

[20] W. Su, C. Mariotti, B. S. Cook, S. Lim, L. Roselli, and M. M. Tentzeris, "A metamaterial-inspired temperature stable inkjet-printed microfluidictunable bandstop filter," in Proc. 44th Eur. Microw. Conf. (EuMC), Rome, Italy, 2014, pp. 9-12.

[21] P. Kabacik and M. E. Bialkowski, "The temperture dependence of substrate parameters and their effect on microstrip antenna performance," IEEE Trans. Antennas Propag., vol. 47, no. 6, pp. 1042-1049, Jun. 1999.

[22] M. Xu, E. M. Eyring, and S. Petrucci, "Dielectric relaxation of chloroform and chloroform-cyclohexane mixtures at gigahertz and terahertz frequencies. The inertial term," J. Molecular Liquids, vols. 73-74, pp. 41-48, Nov. 1997.

[23] F. Buckley and A. A. Maryott, Tables of Dielectric Dispersion Data for Pure Liquids and Dilute Solutions: National Bureau of Standards Circular, vol. 589. Nat. Bureau Standards, Nov. 1958, p. 6.

[24] G. L. Matthaei, L. Young, and E. M. T. Jones, Microwave Filters, Impendence-Matching Networks and Coupling Structures. Norwood, MA, USA: Artech House, 1980.

[25] M. H. Zarifi and M. Daneshmand, "Non-contact liquid sensing using high resolution microwave microstrip resonator," in IEEE MTT-S Int. Microw. Symp. Dig., Phoenix, AR, USA, May 2015, pp. 1-4.

[26] U. Kaatze, "Complex permittivity of water as a function of frequency and temperature," J. Chem. Eng. Data, vol. 34, no. 4, pp. 371-374, Oct. 1989.

[27] D. Slocombe, A. Porch, E. Bustarret, and O. A. Williams, "Microwave properties of nanodiamond particles," Appl. Phys. Lett., vol. 102, no. 24, Jun. 2013, Art. no. 244102.

[28] I. C. Hunter, "Design of contiguous RF filterbanks with application in channelised receivers," IEE Proc. F-Radar Signal Process., vol. 138, no. 3, pp. 289-292, Jun. 1991.

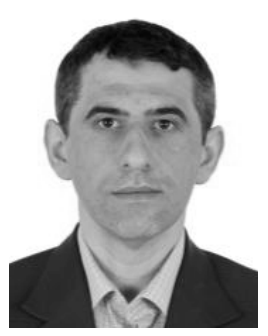

Ali A. Abduljabar received the B.Sc. and M.Sc. degrees in electrical engineering from the University of Basrah, Basrah, Iraq, and the Ph.D. degree in electrical engineering from Cardiff University, Cardiff, U.K.

$\mathrm{He}$ is currently a Lecturer in microwave engineering with the University of Basrah. His current research interests include the design of microwave sensors for microfluidic systems and noninvasive applications and microwave heating techniques.

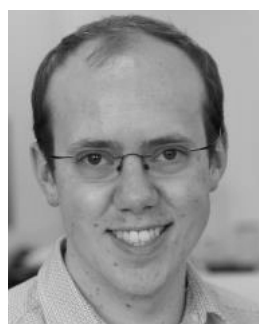

Nicholas Clark received the B.Eng. (Hons.) degree in computer systems engineering from Cardiff University, Cardiff, U.K., in 2013, where he is currently pursuing the $\mathrm{Ph} . \mathrm{D}$. degree.

$\mathrm{He}$ is currently concerned with the application of microwave resonant structures. His current research interests include the characterization and heating of metal powders and structures. 


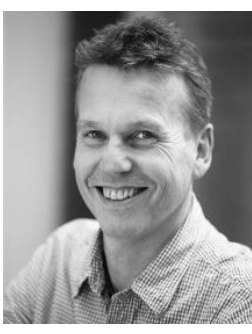

Jonathan Lees received the M.Sc. and Ph.D. degrees in electronic engineering from Cardiff University, Cardiff, U.K.

He is currently a Senior Lecturer with Cardiff University, and a member of the Center for High Frequency Engineering. In recent years, he has applied his microwave expertise to medical applications, including the design of highly integrated microwave PAs for diagnostic applications and the generation and use of high-power pulsed microwave energy for the detection of bacterial DNA. His current research interests include the nonlinear characterization and optimization of highefficiency power, which culminated in the first published $\mathrm{GaN}$ Doherty amplifier.

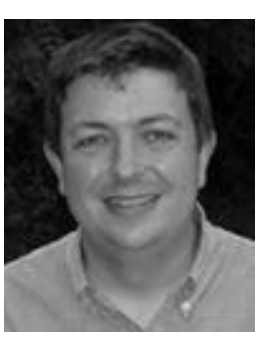

Adrian Porch received the M.A. degree in physics and Ph.D. degree in low-temperature physics from Cambridge University, Cambridge, U.K.

$\mathrm{He}$ is currently a Professor with the School of Engineering, Cardiff University, Cardiff, U.K., and a member of the Centre for High Frequency Engineering. He has over 30 years of experience in applying microwave methods to measure and understand the fundamental properties of electronic materials. More recently, his techniques have been used to develop new types of electromagnetic sensors, with emphasis on applications across different disciplines. 